\title{
KNOW THE STAR, KNOW THE PLANET. III. DISCOVERY OF LATE-TYPE COMPANIONS TO TWO EXOPLANET HOST STARS
}

\author{
Lewis C. Roberts Jr. ${ }^{1}$, Andrei Tokovinin ${ }^{2}$, Brian D. Mason ${ }^{3}$, Reed L. Riddle ${ }^{4}$, William I. Hartkopf ${ }^{3}$, \\ Nicholas M. LaW ${ }^{5}$, and Christoph Baranec ${ }^{6}$ \\ ${ }^{1}$ Jet Propulsion Laboratory, California Institute of Technology, 4800 Oak Grove Drive, Pasadena CA 91109, USA; lewis.c.roberts@jpl.nasa.gov \\ ${ }^{2}$ Cerro Tololo Inter-American Observatory, Casilla 603, La Serena, Chile \\ ${ }^{3}$ U.S. Naval Observatory, 3450 Massachusetts Avenue, NW, Washington, DC 20392-5420, USA \\ ${ }^{4}$ Division of Physics, Mathematics, and Astronomy, California Institute of Technology, Pasadena, CA 91125, USA \\ ${ }^{5}$ Department of Physics and Astronomy, University of North Carolina at Chapel Hill, Chapel Hill, NC 27599-3255, USA \\ Institute for Astronomy, University of Hawaií at Mānoa, Hilo, HI 96720-2700, USA \\ Received 2014 November 21; accepted 2015 February 5; published 2015 March 4
}

\begin{abstract}
We discuss two multiple star systems that host known exoplanets: HD 2638 and 30 Ari B. Adaptive optics imagery revealed an additional stellar companion to both stars. We collected multi-epoch images of the systems with RoboAO and the PALM-3000 adaptive optics systems at Palomar Observatory and provide relative photometry and astrometry. The astrometry indicates that the companions share common proper motion with their respective primaries. Both of the new companions have projected separations less than $30 \mathrm{AU}$ from the exoplanet host star. Using the projected separations to compute orbital periods of the new stellar companions, HD 2638 has a period of $130 \mathrm{yr}$ and 30 Ari B has a period of $80 \mathrm{yr}$. Previous studies have shown that the true period is most likely within a factor of three of these estimated values. The additional component to 30 Ari makes it the second confirmed quadruple system known to host an exoplanet. HD 2638 hosts a hot Jupiter and the discovery of a new companion strengthens the connection between hot Jupiters and binary stars. We place the systems on a color-magnitude diagram and derive masses for the companions which turn out to be roughly 0.5 solar mass stars.
\end{abstract}

Key words: binaries: visual - instrumentation: adaptive optics - stars: individual (HD 2638, 30 Ari B) - stars: solar-type

\section{INTRODUCTION}

Since the discovery of the first exoplanet system in the 1990s, planetary systems have been found in an increasingly varied array of architectures. With this variety has come an increasing number of models and theories to explain these systems. It has also been realized that stellar companions play a key role in the evolution of the planetary dynamics for some systems. There are several proposed ways in which binary stars can influence the orbital properties of exoplanets.

One of these is Kozai migration (Wu \& Murray 2003; Fabrycky \& Tremaine 2007; Wu et al. 2007; Takeda et al. 2008) where the mutual torques between the binary and the planet transfer angular momentum, causing the orbit of the planet to become more eccentric. Eventually the eccentricity becomes high enough that the planet approaches the star, which then tidally circularizes the orbit. Not all exoplanets in binary systems undergo Kozai migration, as it depends on the mutual inclination of the orbits as well as the architecture of the planetary system. Naoz et al. (2012) found that it can account for about $30 \%$ of the observed hot Jupiter planets, which matches well with the projected spin-orbit angle distribution of hot Jupiters.

The stellar companion can also interact with the protoplanetary disk. Rafikov (2013) proposed that the stellar companion affects the protoplanetary disk by slowing the planetesimals in the disk and allowing the formation of planets. This requires a relatively massive disk, but can produce systems such as $\gamma$ Cep and $\alpha$ Cen (Rafikov 2013).

Kley \& Nelson (2008) suggest an alternative method in which an eccentric stellar companion leads to a strong periodic disturbance in the disk whenever the companion is at periastron. This leads to an eccentric disk that changes over time due to the interactions with the stellar companion. This can produce massive eccentric planets, depending on the initial position of the planetesimal. Their modeling did not consider the initial creation of the planetesimals.

Circumbinary planets have also been discovered, where the planet orbits both stars in a short-period orbit, on the order of tens of days (Lee et al. 2009; Doyle et al. 2011). In these systems the planet(s) and the stellar companion are all coplanar implying that they formed from flat circumbinary disks. Since their discovery there has been considerable effort to model the formation and dynamics of these systems (Armstrong et al. 2014; Kley \& Haghighipour 2014).

In the case of multiple stellar systems, coplanar systems are rare. Of the 2413 systems cataloged in the Sixth Catalog of Orbits of Visual Binary Stars (Hartkopf et al. 2001) 48 systems have orbit solutions with all seven Campbell elements for both inner and outer systems in hierarchical arrangements. Of these, only four have the possibility of being coplanar according to the precepts of Fekel (1981).

The various models of exoplanet formation in binary systems can only be fully tested when there are sufficient numbers of known systems, especially systems with relatively short binary periods. There have been a number of surveys to measure the duplicity rates among the exoplanet hosts (Patience et al. 2002; Eggenberger et al. 2007; Chauvin et al. 2011; Mason et al. 2011; Roberts et al. 2011; Ginski et al. 2012). These surveys have revealed several interesting features.

The frequencies of exoplanets among single stars and components of wide binaries (semimajor axis larger than 100 AU) are indistinguishable (Raghavan et al. 2006; Bonavita \& Desidera 2007). Also, the population of exoplanets in wide 
binaries is essentially the same as the population around single stars, indicating that wider binaries have minimal impact on dynamics of the exoplanets (Bonavita \& Desidera 2007). The population of exoplanets in binaries with semimajor axes smaller than $100 \mathrm{AU}$ is statistically different than those orbiting single stars (Zucker \& Mazeh 2002; Bonavita \& Desidera 2007). There are fewer exoplanets in tight stellar binary systems, but the exoplanets tend to be more massive (Desidera \& Barbieri 2007; Duchêne 2010). No planet has been detected in stellar binaries that have a separation of less than $10 \mathrm{AU}$ (Roell et al. 2012). That same paper also determined that multiexoplanet systems have only been detected in binary stars with a projected separation larger than $100 \mathrm{AU}$.

Known close visual binaries are traditionally excluded from radial-velocity (RV) exoplanet programs because the presence of a visual companion degrades the RV precision. This intrinsic bias complicates statistical inferences about exoplanets in binaries. Moreover, a faint visual companion that is itself a close (spectroscopic) binary pair can produce periodic lowamplitude RV modulation in the combined light that can be mistaken for an exoplanet. False positive exoplanet detections caused by unrecognized hierarchical multiplicity of their hosts may reach 1-2\% (Tokovinin 2014b) of the total exoplanet sample. This is yet another reason to observe exo-hosts with high angular resolution and deep dynamical range.

In addition to dynamical interactions, exoplanets and their host stars share a common origin, and determining the properties of that local environment for the star leads to information about the exoplanet. Examples of this include metallicity correlations (e.g., 16 Cyg AB, Schuler et al. 2011), stellar rotation, and activity.

This paper details two multiple star systems that host known exoplanets: HD 2638 and 30 Ari B. Additional stellar components were found in each of these systems by Riddle et al. (2015). This paper discusses the systems and those newly discovered components systems in depth. The observations of the systems are covered in Section 2. Section 3 discusses the HD 2638 system and Section 4 discusses the 30 Ari system. Our results are summarized in Section 5.

\section{OBSERVATIONS}

\subsection{ROBO-AO}

The companions to HD 2638 and 30 Ari B were first detected using the Robo-AO system as part of a survey of nearby binaries with solar type components (Riddle et al. 2015). We have copied the astrometry and photometry from that paper in order to do a more in-depth analysis of these two stars. For those unfamiliar with Robo-AO, we provide a brief introduction to the system. Robo-AO is a robotic laser guide star adaptive optics (AO) system (Baranec et al. 2013, 2014) that operates automatically in order to carry out high efficiency observing programs. Robo-AO is currently deployed on the Palomar Observatory 60 inch telescope. RoboAO uses Rayleigh scattering from a UV laser focused at $10 \mathrm{~km}$ from the telescope as the wavefront reference, and generates diffraction limited images with Strehl ratios of 4-26\% in the $i$ filter. The AO system corrects the high order wavefront aberrations with automated software that operates at a rate of $1.2 \mathrm{kHz}$, sharpening the instantaneous point spread function across the science camera field of view (FOV). A bright star within the FOV is still required to correct the tip-tilt motion, which is corrected by the automated data processing software.

The Robo-AO science camera is an electron multiplying CCD $($ EMCCD) detector with $1024 \times 1024$ pixels; the pixel scale is $43.1 \mathrm{mas} /$ pixel, with a total FOV of $44^{\prime \prime} \times 44^{\prime \prime}$. The EMCCD is operated at 8.6 frames per second in science mode, with an exposure time of $0.115 \mathrm{~s}$ each. A total of 516 frames are gathered during each $60 \mathrm{~s}$ exposure, which are then combined into a single image for further analysis by the automated data processing software. Both targets were observed with the SDSS $i^{\prime}$ filter (York et al. 2000), and additionally HD 2638 was observed with the SDSS $r^{\prime}$ and $z^{\prime}$ bands. The data were collected during multiple observing runs in 2012 and 2013. See Sections 3 and 4 for details. The RoboAO data are procesed by the standard pipeline, which is discussed in Riddle et al. (2015). That paper did not publish error bars for the astrometry measured using speckle processing. It did give mean errors on measurements of control binaries. These companions are fainter than the control binaries, so to be conservative we have set the error bars as 10 mas, which translates to 1.1 in position angle at 0.5 separation.

\subsection{PALM-3000 Adaptive Optics}

In order to confirm and further characterize the companions, we observed both stars on 2013 September 28 UT with the Palomar Observatory Hale $5 \mathrm{~m}$ telescope using the PALM3000 AO system and the PHARO near-IR camera. The PALM3000 AO system is a natural guide star system using two deformable mirrors (Dekany et al. 2013). One corrects lowamplitude high spatial frequency aberrations, while the other corrects the higher-amplitude low spatial frequency aberrations. It is optimized for high contrast observations and routinely produces Strehl ratios greater than $80 \%$ in the Ks band. The PHARO camera uses a HgCdTe HAWAII detector for observations between 1 and $2.5 \mu$ m wavelength (Hayward et al. 2001). The camera has multiple filters in two filter wheels. For HD 2683 we collected 100 frames in both the $J$ and $K s$ filters, while we collected 50 frames of 30 Ari B in the same filters. After debiasing, flat fielding, bad pixel correction, and background subtraction, the frames were coadded. After the individual frames were calibrated, we created multiple images by coadding 10 frames into each image. The iterative blind deconvolution algorithm, fitstars, was used to measure the astrometry and photometry of the objects (ten Brummelaar et al. 1996, 2000).

Photometric error bars were assigned using the technique described in Roberts et al. (2005). For the astrometric error analysis, we computed the standard deviation of the astrometric measurements. Analysis of simulated binaries created using the technique of Roberts et al. (2005) showed that this error is almost entirely measurement error. The other major sources of astrometric error are the errors in the calibration of the plate scale and the position angle offset. The PHARO pixel scale has been measured many times with a combination of an astrometric mask and binary star measurements (Metchev 2006). The changes between observing runs are small. On the night these data were acquired, there was not enough time to repeat the lengthly astrometric mask calibration process, but we observed six calibration binaries and updated the plate scale and the orientation angle offset. This resulted in a plate scale of $0.025 \pm 0.0005$ and a position angle offset of 
Table 1

Basic Information on Two Multiple Systems

\begin{tabular}{|c|c|c|c|c|c|c|}
\hline Object & $\begin{array}{c}\text { WDS } \\
\text { (J2000) }\end{array}$ & HD & HIP & $\begin{array}{c}\pi_{\mathrm{HIP}} \\
(\mathrm{mas})\end{array}$ & $\begin{array}{c}V \\
(\mathrm{mag})\end{array}$ & $\begin{array}{c}\text { Spectral } \\
\text { Type }\end{array}$ \\
\hline HD $2567 \mathrm{~A}$ & 00293-0555 & 2567 & 2292 & $17.63 \pm 0.79$ & 7.76 & G0 \\
\hline 30 Ari A & $02370+2439$ & 16246 & 12189 & $23.93 \pm 0.59$ & 6.48 & F5V \\
\hline 30 Ari B & $\ldots$ & 16232 & 12184 & $24.52 \pm 0.68$ & 7.09 & F6V \\
\hline
\end{tabular}

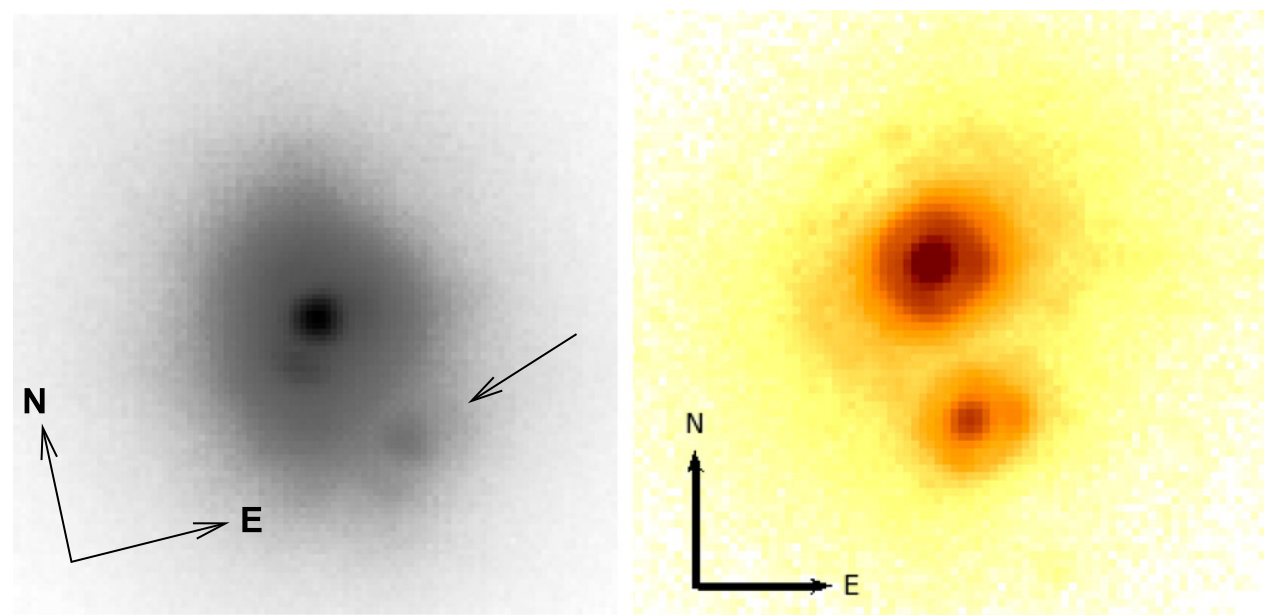

Figure 1. On the left is an $i$ image of the HD 2638 BC from Robo-AO. It is a subimage of the full $44^{\prime \prime}$ field of view and is $\sim 2$ ". 5 across. An arrow points to the location of the newly discovered companion. On the right is a $K s$ image of the HD 2638 BC acquired with the PALM 3000 AO system. It is a subimage of the full $25^{\prime \prime}$ field of view and is $\sim 2^{\prime \prime}$ across.

$0.7 \pm 0.5$. The final error bar on the astrometry was the root sum square of the calibration errors and the scatter in the astrometric measurements.

\section{HD 2638}

HD 2638 (HIP $2350=$ WDS 00293-0555) is a nearby solar type star with an estimated age of $3 \mathrm{Gyr}$ (Moutou et al. 2005). It is paired with HD 2567 (HIP 2292), another solar type star, in a wide $\left(839^{\prime \prime}\right)$ common-proper-motion binary. Shaya \& Olling (2011) determined that the pair had a 99\% chance of being true companions through a Bayesian analysis of the measured astrometry. The astronomical details of the HD 2638 system are given in Table 1 . The measured parallaxes from Hipparcos of the two stars are slightly different. For HD 2638 it is $20.03 \pm 1.49$ mas and for HD 2567 it is $17.63 \pm 0.79$ mas (van Leeuwen 2007). If the parallaxes are correct, it would mean that the stars are separated by $6.8 \mathrm{pc}$, which would mean they are not physically bound. This would disagree with the conclusion of Shaya \& Olling (2011). Independent confirmation that $\mathrm{A}$ and $\mathrm{B}$ are physical comes from their measured RVs being almost identical. $+9.60 \pm 0.3 \mathrm{~km} \mathrm{~s}^{-1}$ for A (Nordström et al. 2004), and $+9.61 \pm 0.01$ for B (Latham et al. 2002). For the distance to the system, we will use the value for HD 2567 since it has the lower error bar. Unresolved binary stars, such as turns out to be the case with HD 2638 (see below), can cause errors in parallaxes and proper motions measured by Hipparcos due to orbital motion in the case of close pairs (Shatskii \& Tokovinin 1998) and due to a cross-talk with the scanning law for wider pairs (Tokovinin et al. 2013). This most likely explains the difference.

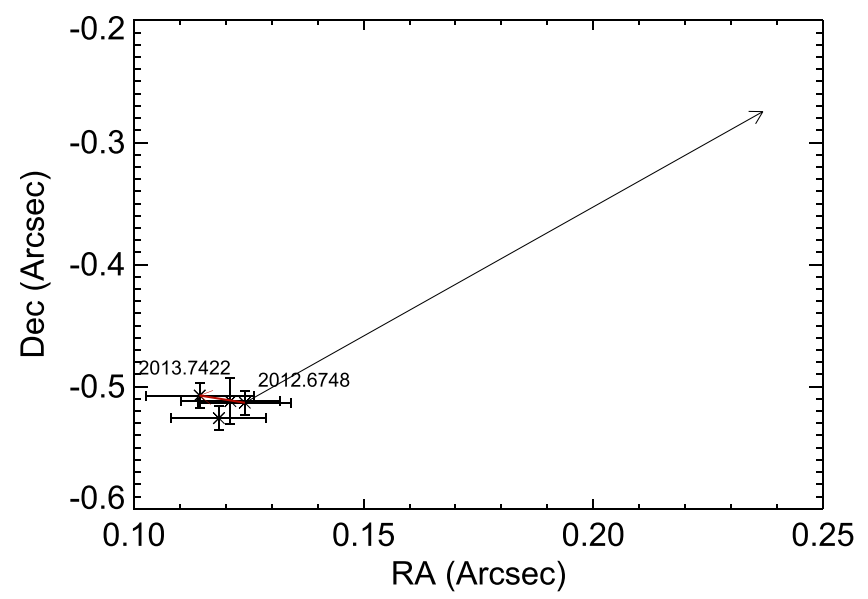

Figure 2. Measured astrometry for HD 2638 BC is plotted. The black arrow is the position the companion would have been at our last observation if it was a background star with no proper motion. The red line shows the actual motion between our first and last observation.

RV observations detected a hot Jupiter orbiting HD 2638 (Moutou et al. 2005). The planet, HD 2638b, has a minimum mass of $0.48 M_{\text {Jup }}$ and an orbital period of $3.4442 \pm 0.0002 \mathrm{~d}$. The orbital semimajor axis is $0.044 \mathrm{AU}$ and the orbit has been tidally circularized. The planet's equilibrium temperature is on the order of $1100 \mathrm{~K}$ (with an assumed albedo of 0.3).

Riddle et al. (2015) imaged a companion to HD 2638 on 2012 September 3 UT. Over the next 13 months, two more observations were carried out with Robo-AO (Riddle et al. 2015) and another observation was made with the $5 \mathrm{~m}$ Hale telescope using the Palm 3000 AO system and the near-IR 
Table 2

Relative Astrometry and Photometry of HD 2638 BC

\begin{tabular}{|c|c|c|c|c|c|c|c|c|}
\hline Epoch & $\theta\left({ }^{\circ}\right)$ & $\rho\left({ }^{\prime \prime}\right)$ & $\Delta r$ & $\Delta i$ & $\Delta z$ & $\Delta J$ & $\Delta K s$ & Instrument \\
\hline 2012.6748 & $166.4 \pm 1.1$ & $0.528 \pm 0.010$ & $\ldots$ & 3.38 & $\ldots$ & $\ldots$ & $\ldots$ & Robo-AO \\
\hline 2012.7621 & $168.7 \pm 1.1$ & $0.553 \pm 0.010$ & 3.87 & $\ldots$ & 3.06 & $\ldots$ & $\ldots$ & Robo-AO \\
\hline 2013.6208 & $166.7 \pm 1.1$ & $0.526 \pm 0.019$ & $\ldots$ & 3.19 & $\ldots$ & $\ldots$ & $\ldots$ & Robo-AO \\
\hline 2013.7422 & $167.3 \pm 1.4$ & $0.52 \pm 0.01$ & $\ldots$ & $\ldots$ & $\ldots$ & $2.50 \pm 0.09$ & $1.99 \pm 0.05$ & PHARO \\
\hline
\end{tabular}

Table 3

Photometry of the New Pairs

\begin{tabular}{|c|c|c|c|c|}
\hline & $\begin{array}{c}i \\
(\mathrm{mag})\end{array}$ & $\begin{array}{c}J \\
(\mathrm{mag})\end{array}$ & $\begin{array}{c}K s \\
(\mathrm{mag})\end{array}$ & $\begin{array}{l}\text { Mass } \\
\left(\mathcal{M}_{\odot}\right)\end{array}$ \\
\hline \multicolumn{5}{|c|}{ HD $2638 \mathrm{BC}, m-M=3.77 \mathrm{mag}$} \\
\hline BC combined & 8.91 & 6.08 & 5.82 & $\ldots$ \\
\hline $\mathrm{BC} \Delta \mathrm{m}$ & 3.29 & 2.50 & 1.99 & $\ldots$ \\
\hline $\mathrm{B}$ & 8.96 & 7.96 & 7.47 & 0.87 \\
\hline $\mathrm{C}$ & 12.25 & 10.46 & 9.46 & 0.46 \\
\hline \multicolumn{5}{|c|}{30 Ari BC, $m-M=3.05 \mathrm{mag}$} \\
\hline BC combined & 6.88 & 6.08 & 5.82 & $\ldots$ \\
\hline $\mathrm{BC} \Delta \mathrm{m}$ & 4.31 & 3.44 & 2.84 & $\ldots$ \\
\hline $\mathrm{B}$ & 6.90 & 6.13 & 5.90 & 1.11 \\
\hline $\mathrm{C}$ & 11.20 & 9.57 & 8.74 & 0.50 \\
\hline
\end{tabular}

PHARO camera. Images of HD $2638 \mathrm{BC}$ are presented in Figure 1 . The measured astrometry and photometry are shown in Table 2. In Riddle et al. (2015) there are two measurements from 2012.7621. One has a position angle of $167: 3$ and the other is 170.0 . The later measurement clearly is in error, as it does not fit the progression of the astrometry. We have chosen to ignore that astrometric point.

HD 2638 has a proper motion of $0.247 \pm 0.0012^{\prime \prime} \mathrm{yr}^{-1}$ ( van Leeuwen 2007). We compare this to the relative motion of the companion to the primary of $0.0108 \pm 0.0175^{\prime \prime} \mathrm{yr}^{-1}$. It is also in a different direction as shown in Figure 2. While the error bar on the relative motion is high compared to the motion, both are much smaller than the proper motion and we conclude that the companion has common proper motion with HD 2638. The measured motion is consistent with orbital motion in that both the position angle and separation are changing in a regular manner within the error bars. With only four data points points it is impossible to determine if there is a deviation from linear motion, which would be required to confirm orbital motion. The binary pair is designated HD 2638 BC. Normally the pair would be designated HD $2638 \mathrm{Ba}, \mathrm{Bb}$, but that could cause confusion with the planetary companion.

\subsection{Discussion}

The components of the newly discovered binary system can be placed on the color-magnitude diagram (CMD), using the photometry in the combined light from the literature and the differential photometry we measured with Robo-AO and PHARO. We also use the the Dartmouth isochrones for $1 \mathrm{Gyr}$ and $3 \mathrm{Gyr}$ age with solar metallicity (Dotter et al. 2008). ${ }^{7}$ The isochrones define the relations between various colors and between mass and absolute magnitude. The combined $J$ and $K$ magnitudes are taken from 2MASS, but the combined SDSS $i$ magnitudes for our targets are not measured directly. We

\footnotetext{
http://stellar.dartmouth.edu/models/webtools.html
}

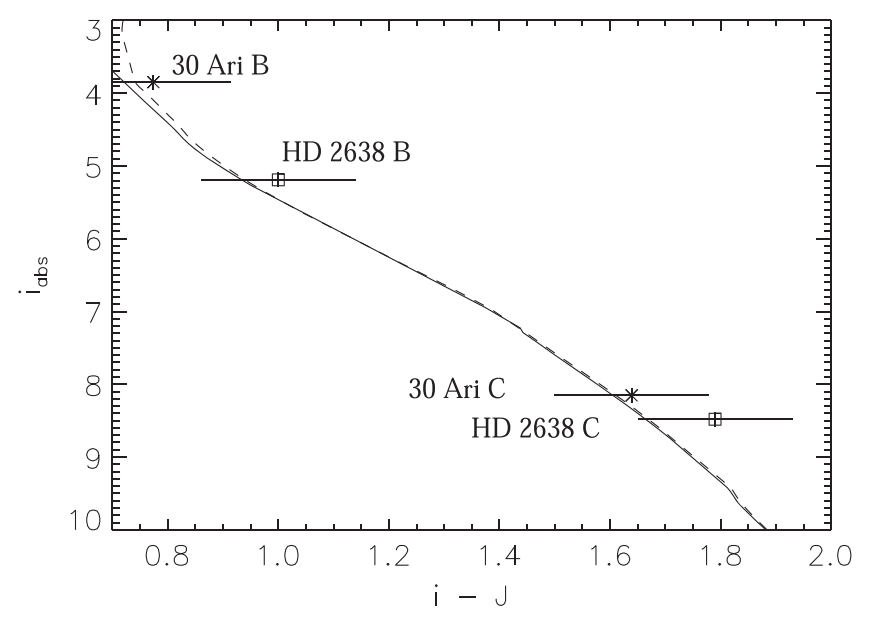

Figure 3. Position of the binary components in the color-magnitude diagram. The full line shows a $1 \mathrm{Gyr}$ isochrone (correspondong to 30 Ari B) and the dashed line shows the $3 \mathrm{Gyr}$ isochrone (corresponding to HD 2638), both with solar metallicity (Dotter et al. 2008).

determined $i$ by extrapolating from the known $V$ and $J$ magnitudes, using polynomial relations between the $V-i$, $i-J$, and $V-K$ colors derived from the Dartmouth isochrones. The combined, differential, and individual magnitudes of the components are listed in Table 3.

Figure 3 shows the position of the binary components in the $(i, i-J) \mathrm{CMD}$, together with the $3 \mathrm{Gyr}$ isochrone defining the main sequence. The masses in Table 3 are determined from the isochrone and the absolute magnitudes in the $i$ band.

We estimate the orbital period, $P$, of the binary from the measured separations, $\rho$, distances, and mass sum $\mathcal{M}$ using Kepler's Third Law, $\frac{a^{3}}{P^{2}}=\mathcal{M}$. The median ratio between the projected separation, $d\left(=\frac{\rho}{\pi_{\mathrm{HIP}}}\right)$, and orbital semimajor axis, $a$, is close to one (Tokovinin 2014a), with scatter by a factor of two caused by orbital phase, orbit orientation, and eccentricity. The strict lower limit is $a>\frac{d}{2}$. Statistical period estimates using the assumption $a=d$ are denoted as $P^{*}$.

The masses of HD $2638 \mathrm{~B}$ and C estimated from their absolute $i^{\prime}$ magnitudes correspond to the spectral types G8V and M1V. The projected separation of $28.5 \mathrm{AU}$ leads to $P^{*} \sim 130 \mathrm{yr}$. The true period likely equals $P^{*}$ to within a factor of three. There is however a small probability that the true separation is much larger than the projected separation, in which case the actual period can be substantially longer than $P^{*}$.

The projected separation of HD $2638 \mathrm{~B}$ and C is quite close. The stellar companion has most likely had a significant impact on the dynamical evolution of the planet. It has long been thought that hot Jupiters formed beyond the snow line and migrated to their current locations. There are a number of 
theories on what caused that migration. One suggested mechanism is that interaction between the protoplanetary disk and the planet causes the planet to migrate inwards (Lin et al. 1996). A second method is planet-planet scattering that puts exoplanets into eccentric orbits that are then tidally circularized (Chatterjee et al. 2008). Another method is Kozai migration, where a stellar companion on an inclined orbit induces exoplanets into eccentric orbits. The primary star will then tidally circularize the planet (Wu \& Murray 2003). Nagasawa et al. (2008) suggested that planet-planet scattering could lead to eccentric inclined exoplanets, which would then start Kozai cycles with the other exoplanets in the system resulting in hot Jupiters. Wu \& Lithwick (2011) proposed that secular chaos could cause the innermost planet to lose angular momentum and then be tidally circularized, forming a hot Jupiter. It is quite probable that all of these methods occur in nature, but we do not know which is the dominant mechanism.

The presence of a stellar companion to HD 2638 does not help us differentiate between these methods. While Kozai migration requires a third body in the system, the other methods are not ruled out by the existence of a stellar companion. To gain a deeper understanding of the dynamical evolution of this system we will need to monitor the system to eventually compute an orbit of the stellar system. Also continued RV monitoring is needed to identify any additional planets in long periods or highly inclined orbits. Continued monitoring would allow measurement of the RV signature of the companion star, which will allow a more complete orbit to be determined.

It is conceivable that the newly discovered component, $\mathrm{C}$, is a close binary masquerading as an exo-planet. We estimate that $\mathrm{C}$ is fainter than $\mathrm{B}$ by about $4 \mathrm{mag}$ in the visible, so the observed RV amplitude of $67.4 \mathrm{~m} \mathrm{~s}^{-1}$ in the combined light of $\mathrm{B}+\mathrm{C}$ corresponds to the $2.7 \mathrm{~km} \mathrm{~s}^{-1}$ amplitude in the velocity of $\mathrm{C}$. The minimum mass of the hypothetic binary companion $\mathrm{Cb}$ is then 0.012 solar, so it could be a $0.1 \mathcal{M}_{\odot}$ dwarf in a $3.44-$ day orbit with a $30^{\circ}$ inclination. This is possible, although statistically unlikely (probability 0.13 ). If, on the other hand, $\mathrm{Ca}$ and $\mathrm{Cb}$ had comparable masses, the $\mathrm{RV}$ amplitude of this putative binary would be about $70 \mathrm{~km} \mathrm{~s}^{-1}$, and an orbital inclination of $\sim 20^{\circ}$ would suffice for blending with the lines of $\mathrm{B}$ and producing the fake exo-planet RV signal. In light of the discovery of $\mathrm{C}$, a more in-depth analysis of the spectra of HD 2638 is desirable to validate the exo-planet.

\section{30 ARI B}

30 Ari B (HIP $12184=$ HD $16232=$ WDS 02370+2439) is a star classified as F6V (Guenther et al. 2009) at a distance of $40.8 \pm \mathrm{pc}$ (van Leeuwen 2007). Along with HIP 12189, it forms the binary system 30 Ari. The pair has a separation of $\sim 40^{\prime \prime}$ and an estimated period of 34,000 years (Tokovinin et al. 2006). Shaya \& Olling (2011) determined that the pair had a near $100 \%$ probability of being a physical system. 30 Ari A (HIP $12189=$ HD 16246) has been listed as an F6III, but Guenther et al. (2009) analyzed its colors and concluded it is actually an F5V star. 30 Ari A is also a spectroscopic binary with a period of 1.109526 days in a nearly circular orbit (Morbey \& Brosterhus 1974). 30 Ari A has a measured age of $0.86 \pm 0.63 \mathrm{Gyr}$ and 30 Ari B has a measured age of $0.91 \pm 0.83$ Gyr (Guenther et al. 2009). Both stars are slightly metal-rich (Guenther et al. 2009). The details of the system are also given in Table 1.
Using the RV technique, Guenther et al. (2009) discovered a $9.88 \pm 0.94 M_{\text {Jup }}$ exoplanet orbiting 30 Ari B with a period of $335.1 \pm 2.5$ days and an eccentricity of $0.289 \pm 0.092$. Based on the star's measured rotational velocity and the average rotational velocity of F-type stars, Guenther et al. (2009) concluded that the star is being observed nearly equator on. Assuming that the planet's orbital axis aligns with the star's spin axis, the minimum mass is close to the true mass. With just over six years of data, there are no indications of additional signals in the RV data coming from additional companions.

Riddle et al. (2015) detected a companion to 30 Ari B with Robo-AO on 2012 September 3 UT at a separation of just over half an arcsecond. The companion was confirmed with RoboAO approximately a year later and we conducted follow up observations in the near-IR with the Palm 3000 AO system on the Palomar $5 \mathrm{~m}$ telescope at approximately the same time. Images of 30 Ari BC are shown in Figure 4. The measured astrometry and photometry are in Table 4 . The error bars of 30 Ari BC are smaller than the error bars of HD 2638 BC because 30 Ari $\mathrm{BC}$ is a brighter star and $\mathrm{AO}$ performance is improved on brighter targets.

30 Ari B has a proper motion of $0.151 \pm 0.00075^{\prime \prime} \mathrm{yr}^{-1}$ (van Leeuwen 2007). Between the first and last observation the companion moved $0.0229 \pm 0.0199^{\prime \prime} \mathrm{yr}^{-1}$. This is illustrated in Figure 5. From this we conclude that the companion has common proper motion with 30 Ari B. Like HD 2638, this would normally be designated 30 Ari $\mathrm{Bb}$, but to avoid confusion with the planet we designate it as 30 Ari C. The measured motion is consistent with orbital motion in that both the position angle and separation are changing in a regular manner. With only three data points it is not possible to determine if there is a deviation from linear motion, which would be required to confirm orbital motion.

As part of a duplicity survey of exoplanet host stars, Ginski et al. (2012) observed 30 Ari B with the $2.2 \mathrm{~m}$ Calar Alto telescope and the AstraLux lucky imaging camera in the SDSS $i$ filter on 2010 February 23. They did not detect the companion due to the limited dynamic range of the lucky imaging system and poor weather.

\subsection{Discussion}

The projected separation between B and C is 22.3 AU and corresponds to the statistical period $P^{*}=80 \mathrm{yr}$. Using the same technique as used for HD 2638, we computed photometry and masses for 30 Ari $\mathrm{BC}$ which are presented in Table 3. The luminosities and masses of $\mathrm{B}$ and $\mathrm{C}$ correspond to spectral types F8V and M1V, respectively.

As in the case of HD $2638 \mathrm{~B}$, the possibility that the exoplanet signal comes from a hierarchical multiple system needs to be addressed. According to (Guenther et al. 2009), the axial rotation of $B$ is $38 \mathrm{~km} \mathrm{~s}^{-1}$, while the $\mathrm{RV}$ amplitude is $0.27 \mathrm{~km} \mathrm{~s}^{-1}$. Although at visible wavelengths $\mathrm{C}$ is fainter than $\mathrm{B}$ by at least $5 \mathrm{mag}$, it has a lower effective temperature and stronger lines, making it difficult to evaluate the amplitude of the hypothetic binary $\mathrm{Ca}, \mathrm{Cb}$ required to mimick the exoplanet. An amplitude of $10 \mathrm{~km} \mathrm{~s}^{-1}$, for example, can be produced by a companion $\mathrm{Cb}$ of $0.26 \mathcal{M}_{\odot}$ at $90^{\circ}$ inclinantion.

Guenther et al. (2009) points out that, "...according to Eggenberger et al. (2007) most of the very massive planets are in binary systems. But ... the distance to 30 Ari $\mathrm{A}$ is presumably too large to have any effect on the properties of 


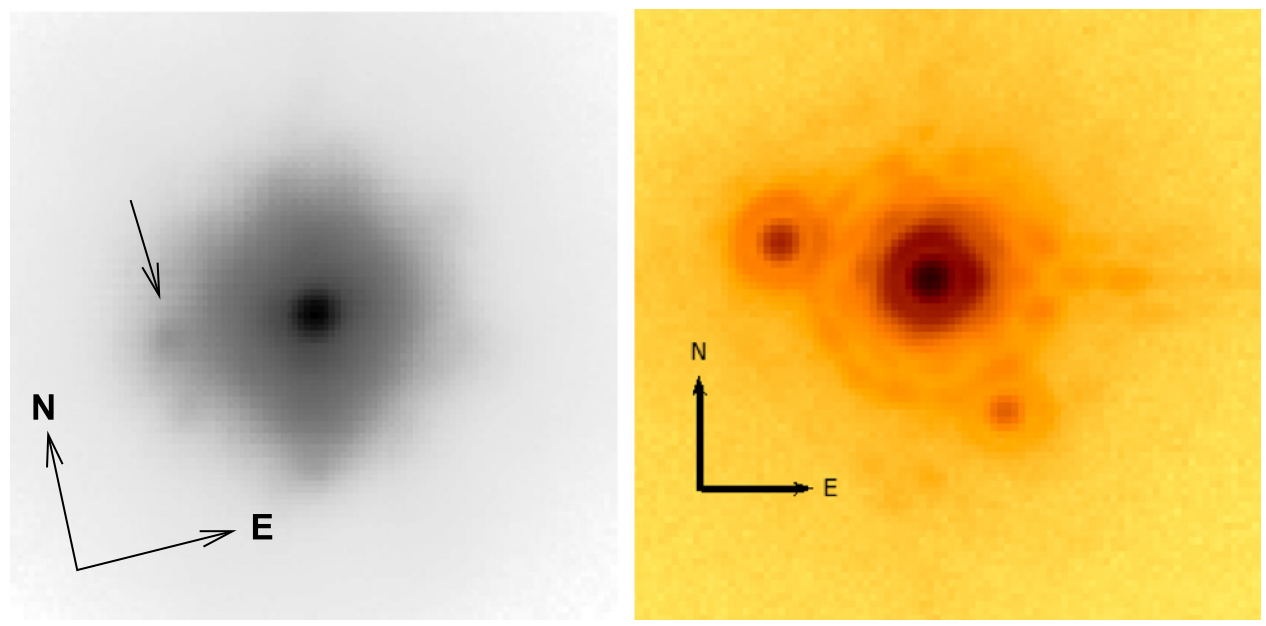

Figure 4. On the left is an $i$ image of 30 Ari BC from Robo-AO. It is a subimage of the full $44^{\prime \prime}$ field of view and is $\sim 2 . " 5$ across. The location of the newly discovered companion is indicated by the arrow. On the right is a $K s$ image of 30 Ari BC acquired with the PALM 3000 AO system. The object to the lower right of the primary star of the image is a ghost image caused by the neutral density filter. This is a subimage of the full $25^{\prime \prime}$ field of view and is $\sim 2^{\prime \prime}$ across.

Table 4

Relative Astrometry and Photometry of 30 Ari B

\begin{tabular}{lccccc}
\hline \hline Epoch & $\theta\left({ }^{\circ}\right)$ & $\rho\left({ }^{\prime \prime}\right)$ & $\Delta i$ & $\Delta J$ & $\Delta K s$ \\
\hline 2012.6750 & $285.9 \pm 1.1$ & $0.528 \pm 0.010$ & $4.32 \pm 0.1$ & $\ldots$ & $\ldots$ \\
2013.6167 & $285.2 \pm 0.2$ & $0.536 \pm 0.002$ & $4.31 \pm 0.1$ & $\ldots$ & $\ldots$ \\
2013.7426 & $284.77 \pm 0.79$ & $0.55 \pm 0.008$ & $\ldots$ & $3.44 \pm 0.15$ & Instrument \\
\hline
\end{tabular}

the planet." Our discovery of 30 Ari C may explain why it is not the exception.

The detection of a stellar companion to 30 Ari B means that the 30 Ari system is a quadruple system containing at least one exoplanet. This makes it the second confirmed quadruple system known to host an exoplanet. The first is KIC 4862625 hosting the planet Ph1b (Schwamb et al. 2013). Ph1b is a circumbinary planet orbiting an eclipsing binary consisting of G- and M-type dwarfs. The other two components of the quadruple are in a tight visual binary with a projected separation of $1000 \mathrm{AU}$ from the G/M pair.

The similarity between KIC 4862625 and 30 Ari is that both systems are quadruples consisting of two relatively close pairs that are widely separated. These $2+2$ systems are relatively common. Riddle et al. (2015) found that over $10 \%$ of wide binaries are actually $2+2$ quadruples and that the majority of quadruples are in this form, rather than the form of a triple system with a distant companion (i.e., $3+1$ ). In both of the quadruple systems hosting exoplanets, the two binaries making up the quadruple are most likely far enough apart that there is no impact on the dynamics or the formation of the exoplanet.

\section{SUMMARY}

We have detected stellar companions to the exoplanet host stars HD 2638 and 30 Ari B. Both systems are part of wider multiple star systems. In the case of 30 Ari B, the other component, 30 Ari $\mathrm{A}$, is also a binary star making it a quadruple system. This is the second quadruple system known to host an exoplanet.

Bonavita \& Desidera (2007) found that binaries with exoplanets tend to have low mass stellar companions. Both of the systems detected here continue that trend. This could be a selection effect, as most RV surveys have eliminated known binaries from their observing lists. These known binaries were mostly discovered by the Hipparcos satellite, visual micrometers, or speckle interferometry instruments, which all have limited dynamic range. AO is currently being used for most exoplanet duplicity searches and it can achieve a much higher dynamic range allowing for the detection of late-type stars.

HD $2638 \mathrm{BC}$ and 30 Ari BC both have projected separations of less than $30 \mathrm{AU}$ and add to the rather small number of systems with such low separations. The stellar companions have the potential to have been a key part of the dynamical evolution of the exoplanets. However, the veracity of these exoplanets has to be addressed in light of the new companions, to eliminate the possibility that these RV discoveries are false positives caused by hierarchical multiplicity. These systems strengthen the link between massive planets and binary stars. The astrometry of these binaries should continue to be monitored. In the near future this will hopefully detect orbital motion and provide an indication to the period of the objects. With a long enough span of observations, an orbit can be computed, which will provide a more complete picture of the nature of the dynamical interaction between the binary companion and the exoplanet. In addition to the system's astrometry, their radial velocities should also be monitored. This may reveal a trend in the RV caused by the stellar companion. Knowledge of the RV trend coupled with measured astrometry will enable the computation of the full orbital solution.

We thank the staff of the Palomar Observatory for their invaluable assistance in collecting these data. This paper was based in part on observations obtained at the Hale Telescope, Palomar Observatory as part of a continuing collaboration between the California Institute of Technology, NASA/JPL, Oxford University, Yale University, and the National 


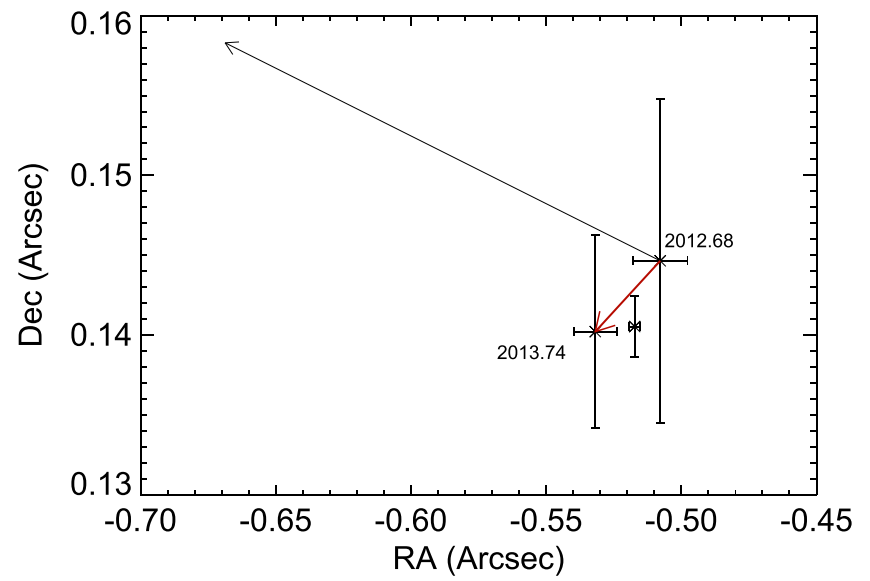

Figure 5. Measured astrometry for 30 Ari BC is plotted. The black arrow is the position the companion would have been at our last observation if it was a background star with no proper motion. The red line shows the actual motion between our first and last observation.

Astronomical Observatories of China. A portion of the research in this paper was carried out at the Jet Propulsion Laboratory, California Institute of Technology, under a contract with the National Aeronautics and Space Administration (NASA). The Robo-AO system is supported by collaborating partner institutions, the California Institute of Technology and the Inter-University Centre for Astronomy and Astrophysics, by the National Science Foundation under Grant Nos. AST0906060, AST-0960343, and AST-1207891, by a grant from the Mt. Cuba Astronomical Foundation and by a gift from Samuel Oschin. C.B. acknowledges support from the Alfred P. Sloan Foundation. This research made use of the Washington Double Star Catalog maintained at the U.S. Naval Observatory, the SIMBAD database, operated by the CDS in Strasbourg, France, and NASA's Astrophysics Data System.

Facilities: PO:1.5 m (Robo-AO), Hale (PHARO)

\section{REFERENCES}

Armstrong, D. J., Osborn, H. P., Brown, D. J. A., et al. 2014, MNRAS, 444, 1873

Baranec, C., Riddle, R., Law, N., et al. 2013, JVE, 72, e50021

Baranec, C., Riddle, R., Law, N., et al. 2014, ApJL, 790, L8

Bonavita, M., \& Desidera, S. 2007, A\&A, 468, 721

Chatterjee, S., Ford, E. B., Matsumura, S., \& Rasio, F. A. 2008, ApJ, 686, 580

Chauvin, G., Beust, H., Lagrange, A.-M., \& Eggenberger, A. 2011, A\&A, 528,8

Dekany, R., Roberts, J., Burruss, R., et al. 2013, ApJ, 776, 130

Desidera, S., \& Barbieri, M. 2007, A\&A, 462, 345
Dotter, A., Chaboyer, B., Jevremović, D., Kostov, V., Baron, E., \& Ferguson, J. W. 2008, ApJS, 178, 89

Doyle, L. R., Carter, J. A., Fabrycky, D. C., et al. 2011, Sci, 333, 1602

Duchêne, G. 2010, ApJL, 709, L114

Eggenberger, P., Udry, S., Chauvin, G., et al. 2007, A\&A, 474, 273

Fabrycky, D., \& Tremaine, S. 2007, ApJ, 669, 1298

Fekel, F. C. 1981, ApJ, 246, 879

Ginski, C., Mugrauer, M., Seeliger, M., \& Eisenbeiss, T. 2012, MNRAS, 421, 2498

Guenther, E. W., Hartmann, M., Esposito, M., et al. 2009, A\&A, 507, 1659

Hartkopf, W. I., Mason, B. D., \& Worley, C. E. 2001, AJ, 122, 3472 (current version available online at http://www.usno.navy.mil/USNO/astrometry/ optical-IR-prod/wds/orb6)

Hayward, T. L., Brandl, B., Pirger, B., et al. 2001, PASP, 113, 105

Kley, W., \& Nelson, R. P. 2008, A\&A, 486, 617

Kley, W., \& Haghighipour, H. 2014, A\&A, 564, A72

Latham, D., Stefanik, R. P., Torres, G., et al. 2002, AJ, 124, 1144

Lee, J. W., Kim, S.-L., Kim, C.-H., et al. 2009, AJ, 137, 3181

Lin, D. N. C., Bodenheimer, P., \& Richardson, D. C. 1996, Natur, 380, 606

Mason, B. D., Hartkopf, W. I., Raghavan, D., et al. 2011, AJ, 142, 176

Metchev, S. A. 2006, Ph.D. thesis, California Institute of Technology

Morbey, C. L., \& Brosterhus, E. B. 1974, PASP, 86, 455

Moutou, C., Mayor, M., Bouchy, F., et al. 2005, A\&A, 439, 367

Nagasawa, M., Ida, S., \& Bessho, T. 2008, ApJ, 678, 498

Naoz, S., Farr, W. M., \& Rasio, F. A. 2012, ApJL, 754, L36

Nordström, B., Mayor, M., Andersen, J., et al. 2004, A\&A, 418, 989

Patience, J., White, R. J., Ghez, A. M., et al. 2002, ApJ, 581, 654

Rafikov, R. R. 2013, ApJL, 765, L8

Raghavan, D., Henry, T. J., Mason, B. D., et al. 2006, ApJ, 646, 523

Riddle, R. L., Tokovinin, A., Mason, B. D., et al. 2015, ApJ, 799, 4

Roberts, L. C., Jr., Turner, N. H., Bradford, L. W., et al. 2005, AJ, 130, 2262

Roberts, L. C., Jr., Turner, N. H., ten Brummelaar, T. A., Mason, B. D., \& Hartkopf, W. I. 2011, AJ, 142, 175

Roell, T., Neuhäuser, R., Seifahrt, A., \& Mugrauer, M. 2012, A\&A, 542 A92

Schuler, S. C., Cunha, K., Smith, V. V., et al. 2011, ApJL, 737, L32

Schwamb, M. E., Orosz, J. A., \& Carter, J. A. 2013, ApJ, 768, 127

Shatskii, N. I., \& Tokovinin, A. A. 1998, AstL, 24, 673

Shaya, E. J., \& Olling, R. P. 2011, ApJS, 192, 2

ten Brummelaar, T. A., Mason, B. D., Bagnuolo, W. G., Jr., et al. 1996, AJ, 112,1180

ten Brummelaar, T. A., Mason McAlister, H. A., Roberts, L. C., Jr., et al. 2000, AJ, 119, 2403

Takeda, G., Kita, R., \& Rasio, F. 2008, ApJ, 683, 1063

Tokovinin, A., Thomas, S., Sterzik, M., \& Udry, S. 2006, A\&A, 450, 681

Tokovinin, A., Mason, B. D., \& Hartkopf, W. I. 2010, AJ, 139, 743

Tokovinin, A., \& Líepine, S. 2012, AJ, 144, 102

Tokovinin, A., Hartung, M., \& Hayward, T. L. 2013, AJ, 146, 8

Tokovinin, A. 2014, AJ, 147, 86

Tokovinin, A. 2014, AJ, 147, 87

van Leeuwen, F. 2007, A\&A, 474, 653

Wu, Y., \& Murray, N. 2003, ApJ, 589, 605

Wu, Y., Murray, N. W., \& Ramasahai, J. M. 2007, ApJ, 670, 820

Wu, Y., \& Lithwick, Y. 2011, ApJ, 735, 109

York, D. G., Adelman, J., Anderson, J. E., Jr., et al. 2000, AJ, 120, 1579

Zucker, S., \& Mazeh, T. 2002, ApJL, 568, L113 